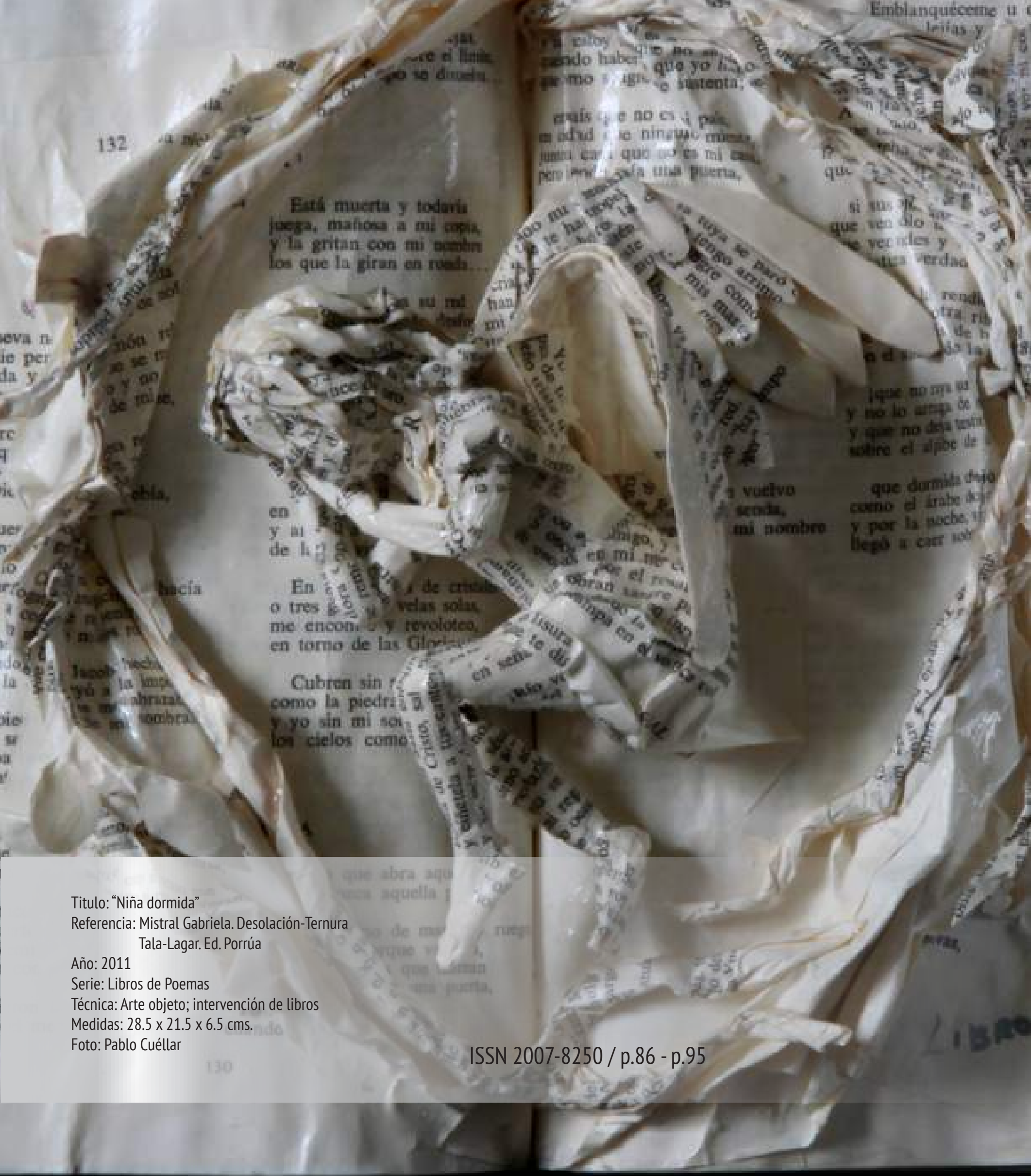




\section{Los estilos de aprendizaje en la resolución de problemas matemáticos}

\section{Learning styles in solving mathematical problems}

\section{Ma. del Socorro Elizondo Treviño}

\section{Resumen}

El objetivo de este proyecto de investigación es identificar el tipo de error en el procesamiento de la información en relación a los estilos de aprendizaje en la resolución de problemas matemáticos. Este estudio tiene un diseño transversal y un alcance explicativo y se realizó en la Preparatoria No.1 de la Universidad Autónoma de Nuevo León (UANL), que cuenta con una población de 2 mil treinta alumnos de tercer semestre, de la cual 340 de ellos son objeto de investigación. A esta muestra de alumnos se les aplicó un instrumento y se presentan los siguientes análisis: de frecuencia y porcentajes, comparativos, correlacionales y análisis factoriales. Las causas que motivan este estudio son los bajos resultados en los exámenes en la Preparatoria y en los exámenes globales en el Nivel Medio Superior (NMS) de la UANL, el desinterés de los estudiantes cuando no pueden resolver problemas y su falta de estrategias de estudio en los alumnos así como la falta de estrategias de los profesores. Los beneficios que se esperan obtener con esta investigación es la disminución del índice de reprobación en matemáticas, la mejora de la práctica educativa para que todos los alumnos tengan interés en la materia, la creación de estrategias que permitan identificar el tipo de error e implementarlas en otras materias. Se concluye que en la resolución de problemas matemáticos existen dificultades en el tipo de error de procesamiento de información en relación a los estilos de aprendizaje.

Palabras clave: resolución, problemas, relación, estilos, aprendizaje, matemáticas.

\begin{abstract}
The objective of this research project is to identify the type of error in the processing of information in relation to learning styles in solving mathematical problems. This study has a transversal design and an explanatory scope and was carried out in the High School No. 1 of Universidad Autónoma de Nuevo Leon (UANL), which has a population of 2 thousand and 30 students in the third semester, of which 340 of them are subject of research, to whom a questionnaire in which results are observed in the following analyzes frequency and percentages, comparative, correlational and factor analysis. The causes that motivate this study are the low results in the examinations in the Preparatory and in the global examinations in the Medium Superior Degree (NMS) of the UANL, the uninterested of the students when they cannot solve problems, the lack of strategies of study in the students, the lack of strategies of the teachers and the benefits that are expected to obtain with this investigation are decrease of the index of failure in mathematics, improve the educational practice so that all the students have interest in the subject, create strategies that allow to identify the type of error and implement them in other matters. It is concluded that in solving mathematical problems there are difficulties in the type of information processing error in relation to learning styles.
\end{abstract}

Keywords: solving, problems, relationship, styles, learning, mathematics. 
$\mathrm{E}$ las propuestas curriculares internacionales así como del país, la importancia de la resolución de problemas matemáticos se ha mantenido e incluso acrecentado; al resolver problemas los estudiantes manifiestan su estilo de aprendizaje; en el caso del Nivel Medio Superior (NMS) los alumnos presentan un estilo de aprendizaje ya conformado durante su historia académica.

Después de un determinado lapso de estudio, algunos tienden a ajustar 0 agregar ciertas estrategias y características de aprendizaje que derivan en un nuevo estilo; otros por el contrario, las mantienen reforzando el estilo preexistente.

La revisión bibliográfica que se incluye en este apartado comprende la resolución de problemas y los estilos de aprendizaje. La resolución ha sido identificada como una actividad importante en el aprendizaje de las matemáticas, y puede abordarse desde diversos contextos; los estudiantes experimentan la utilidad de las Matemáticas en la realidad que les rodea y de la que forman parte.

Al analizar el sentido estructural de 33 estudiantes de bachillerato en España, se manifiesta que al trabajar con expresiones algebraicas simples y complejas, en el contexto de la simplificación de fracciones que involucran las igualdades notables: cuadrado de la suma, cuadrado de la diferencia, diferencia de cuadrados y propiedad distributiva o factor común. Los resultados informan sobre algunas de las dificultades que los estudiantes encuentran en la tarea propuesta para hacer uso de sentido estructural, y sobre la diferente influencia de las características de las expresiones algebraicas en las estrategias empleadas por los estudiantes (Vega-Castro, Molina y Castro, 2012).

Desde una perspectiva sociocultural, la resolución de problemas se utilizó para abordar los cambios que se manifiestan en el discurso matemático de los estudiantes cuando tratan de definir un objeto matemático; en un estudio en el que participaron 13 estudiantes de segundo curso de Bachillerato (edades entre 16 y 18 años) y 38 estudiantes para profesor de primaria (edades entre 18 y 21 años) de escuelas de España.

Se identificaron cambios diferentes en el discurso matemático de los estudiantes, a partir de la caracterización de las relaciones entre narrativas asumidas y rutinas que se han puesto de manifiesto en el discurso de Escudero, Gavilán y Sánchez-Matamoros (2014).

Diversas investigaciones abordan el término discurso matemático desde diferentes perspectivas, que es el que incluye palabras relacionadas con cantidades y figuras o símbolos creados para facilitar esta forma particular de comunicación. De la revisión bibliográfica, se concluye que la resolución de problemas matemáticos es una actividad importante y se debe atender en el proceso de enseñanzaaprendizaje de la matemática.

La teoría de los estilos de aprendizaje tiene una estrecha relación en el proceso de enseñanza-aprendizaje, debe ser incorporada en el trabajo cotidiano de todos los docentes, y las pretensiones de la educación y

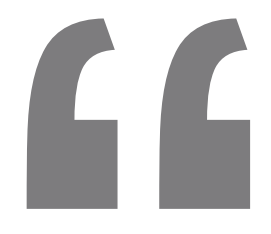

Diversas

investigaciones

abordan desde

diferentes

perspectivas el

término discurso

matemático,

que es el que incluye palabras relacionadas con cantidades y figuras o símbolos creados para facilitar esta forma particular de comunicación". 
los modelos educativos van a verse coronados con efectividad; en esta investigación se pretende diagnosticar los estilos de aprendizaje en la resolución de problemas.

Felder y Silverman (1988) desarrollaron un modelo de los Estilos de Aprendizaje (EA), verifican la validez y confiabilidad del instrumento de estilos de aprendizaje y su relación con edad, género y semestre en curso, el instrumento se aplicó a 93 estudiantes (64 mujeres y 29 hombres), de Ingeniería del Instituto Politécnico Nacional, en México; el modelo clasifica los estilos de aprendizaje en 5 dimensiones:

- sensitivo-intuitiva

- visual-verbal

- activo-reflexivo

- secuencial-global

- inductivo-deductiva

Los resultados obtenidos revelan que los estudiantes manifiestan preferencia en el estilo activo sobre el reflexivo, es decir, tienen una tendencia a realizar actividades físicas y a la experimentación, los alumnos son más sensitivos que intuitivos, los estilos de aprendizaje que adoptan los hombres y las mujeres son los mismos en las dimensiones sensitivo-intuitiva, visual-verbal y secuencial-global (Ocampo, Guzmán, Camarena y De Luna, 2014).

El objetivo general de esta investigación es demostrar la relación entre la resolución de problemas matemáticos y los estilos de aprendizaje de los alumnos del Nivel Medio Superior.

Los objetivos específicos de la investigación son los siguientes: Mostrar

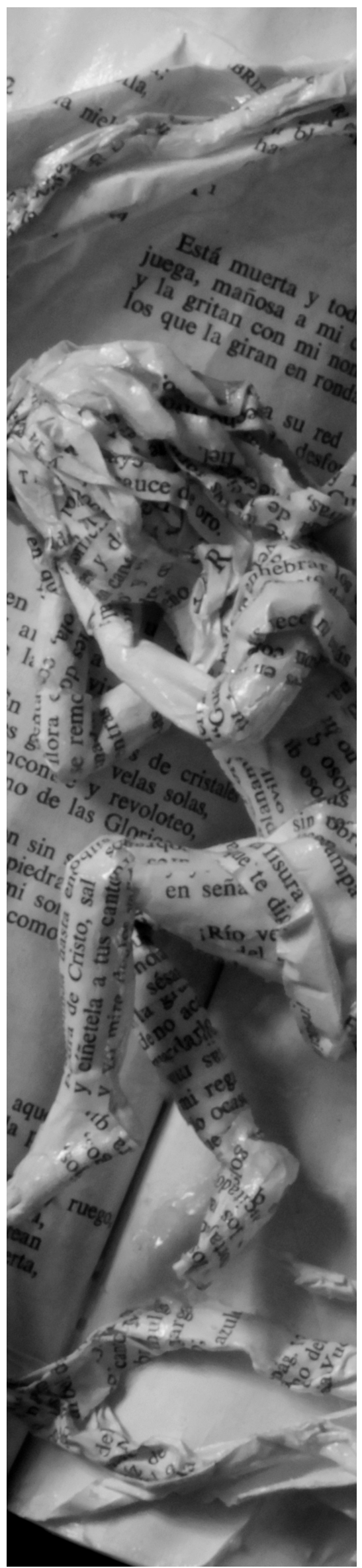

las diferencias significativas en comprender un problema, según el género. Demostrar la relación entre la resolución de problemas y los estilos de aprendizaje.

Una de las metas que persigue este trabajo de investigación es lograr que el alumno adquiera o refuerce ciertas habilidades cognoscitivas que le permitan resolver problemas matemáticos y que posteriormente, pueda transferir el uso de estas habilidades para cualquier disciplina, a sus estudios superiores o a su campo profesional, sin embargo, para que el aprendizaje escolar cumpla la función de ser utilizado en cualquier contexto, es necesario que el individuo no sólo adquiera un conocimiento determinado, sino que además lo reconstruya en situaciones diversas.

La resolución de problemas en general ha recibido distintas definiciones en función de la teoría psicológica que la ha abordado, los teóricos de la Gestalt consideraron que el núcleo de la resolución de problemas consistía en la comprensión del problema como un todo.

Para los teóricos del conductismo, la resolución de problemas matemáticos residía en las conexiones entre las acciones ejecutadas por el sujeto que resuelve el problema y las condiciones bajo las cuales se manifiestan esas acciones.

Cada estudiante posee un estilo de aprendizaje predominante, que el docente lo conozca puede contribuir para que sus estudiantes logren alcanzar un mayor nivel de aprendizaje el cual se ve influido por las características cognitivas de las disciplinas; 
en las matemáticas, sin embargo, éstas no están claramente delimitadas. El desempeño académico de los estudiantes ha sido asociado con muchos factores que rodean las condiciones en que ellos logran el estilo de aprendizaje.

La trascendencia educativa de la expresión estilo estuvo determinada por sus cualidades y alcances conceptuales, esta noción no sólo incluye propiedades de la inteligencia, sino que también pondera otros componentes del aprendizaje; tal es el caso de la percepción de logro, la motivación, el desempeño y el contexto, entre otros (Valadez, 2009).

Los estilos de aprendizaje se entienden como variables personales que, a mitad de camino entre la inteligencia y la personalidad, explican las diferentes formas de abordar, planificar y responder ante las demandas del aprendizaje (Camarero, Martín y Herrero, 2000).

Los Estilos de Aprendizaje son los rasgos cognitivos, afectivos y fisiológicos, que sirven como indicadores relativamente estables, de cómo los discentes perciben, interaccionan y responden a sus ambientes de aprendizaje. (según define Keefe) (Alonso, Gallego y Honey, 1997 p.103 como se citó en Cagliolo, Junco y Peccia, 2010)

Alonso, Gallego y Honey (1997), como se citó en Bolívar y Rojas (2008, pp. 199-215), realizan una propuesta en la cual ubican a los estudiantes en cuatro estilos:

Activos: buscan experiencias nuevas, son de mente abierta, nada escépticos y realizan con entusiasmo las tareas nuevas; son personas que viven en el aquí y el ahora.

Reflexivos: gustan observar las experiencias desde diferentes perspectivas, reúnen datos para analizarlos con detenimiento antes de llegar a alguna conclusión, prefieren ser prudentes y mirar bien antes de actuar.

Teóricos: suelen ser perfeccionistas, por lo general, buscan integrar los hechos en teorías coherentes, gustan de analizar y sintetizar, para ellos, la racionalidad y la objetividad son aspectos prioritarios.

Pragmáticos: su principal característica se relaciona con la aplicación práctica de las ideas, son realistas cuando se trata de tomar una decisión o resolver un problema, su filosofía es: si funciona, es bueno.

La metodología que se utilizó para la realización de este trabajo es la siguiente:

-Definir el enfoque de la investigación.

-Determinar la población y la muestra con la cual se realizó la investigación.

-Diseño del instrumento y su piloteo.

-Selección de la técnica que se utilizó para lograr dar cumplimiento a los objetivos específicos que permitieron alcanzar el objetivo general.

-Análisis de los datos recolectados.

-Presentación de los resultados obtenidos en este estudio.

Para calcular el tamaño de la muestra se utilizó el programa STATS (Sample Size Determination), (Hernández,
Fernández y Baptista, 2014). Tamaño del universo: 2030; Error máximo aceptable: 5\%; Porcentaje estimado de la muestra: $50 \%$; Nivel deseado de confianza: 95\%; El resultado que proporcionó STATS es: 340 alumnos para considerar en la muestra.Se realizó un muestreo probabilístico al azar con los grupos de tercer semestre en la Preparatoria 1 de la UANL y el cuestionario se aplicó a 340 estudiantes, 205 mujeres y 135 varones entre 16 y 17 años de edad.

El cuestionario aplicado consta de 111 preguntas, está dividido en tres bloques, utilizando la escala nominal y ordinal, el primer bloque está compuesto de 13 variables signalíticas, está integrado por variables como sexo, edad, matemáticas, máximo grado de estudios del padre, de la madre, entre otras; los otros dos bloques corresponden a resolución de problemas y estilos de aprendizaje.

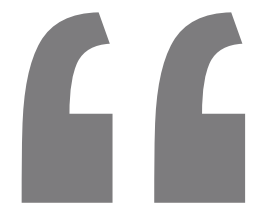

Los Estilos de Aprendizaje son los rasgos cognitivos, afectivosy fisiológicos, que sirven como indicadores". 
Tabla 1

Distribución de la muestra con respecto a la materia que más les gusta

\begin{tabular}{ccc}
\hline Materia & Frecuencia & Porcentaje $^{\mathrm{a}}$ \\
\hline Matemáticas & 64 & 18.8 \\
Literatura & 31 & 9.1 \\
Empresarial & 64 & 18.8 \\
Base de datos & 16 & 4.7 \\
Inglés & 64 & 18.8 \\
Sociales & 41 & 12.1 \\
Física & 20 & 5.9 \\
Ninguna & 33 & 9.7 \\
Todas & 7 & 2.1 \\
\hline Total & 340 & 100.0 \\
\hline
\end{tabular}

Fuente: Elaboración propia; ${ }^{a}$ representa el porcentaje válido para este indicador.

Se observa que las materias que más les gusta a los alumnos son: matemáticas, empresarial e inglés $(n=64)$ y ninguna les gusta $(n=33)$.

Tabla 2

Distribución de la muestra a partir del sexo

\begin{tabular}{ccc}
\hline Sexo & Frecuencia & Porcentaje $^{\text {a }^{2}}$ \\
Mujer & 205 & 60.3 \\
Hombre & 135 & 39.7 \\
\hline Total & 340 & 100.0 \\
\hline
\end{tabular}

Fuente: Elaboración propia; ${ }^{a}$ representa el porcentaje válido para este indicador.

La muestra estuvo compuesta por 340 alumnos de tercer semestre, el $60.3 \%(n=205)$ son mujeres y el $39.7 \% \quad(n=135)$ son hombres, observamos que de los grupos de estudio existe un porcentaje mayor en el sexo femenino.

\section{Análisis comparativo}

Dentro de las variables que miden pensamiento matemático (cognitivo), se presentan diferencias significativas entre mujeres y hombres en lo siguiente:

Tabla 3

Prueba " $\mathrm{t}$ " de student entre sexo y pensamiento matemático (cognitivo)

\begin{tabular}{|c|c|c|c|c|c|}
\hline Variable & $\begin{array}{c}\overline{\mathrm{X}} \\
\text { Femenino }\end{array}$ & $\begin{array}{c}\overline{\mathrm{X}} \\
\text { Masculino }\end{array}$ & $\begin{array}{c}\text { t- } \\
\text { value }\end{array}$ & $\mathrm{df}$ & $\mathrm{p}$ \\
\hline 11.- Lees el enunciado del problema & 8.54 & 7.93 & 2.36 & 338 & 0.01 \\
\hline $\begin{array}{l}\text { 13.- Inviertes mucho tiempo } \\
\text { en comprender el problema }\end{array}$ & 7.03 & 6.28 & 2.54 & 338 & 0.01 \\
\hline
\end{tabular}


En la Tabla 3, se puede observar que tiempo en comprender el problema", las mujeres presentan una media significativamente mayor que los hombres en las variables: "lees el enunciado del problema e inviertes mucho lo que indica que las mujeres a pesar de leer el enunciado del problema tardan más tiempo en la comprensión de él, a diferencia de los hom- bres. Se puede inferir que las mujeres le dedican mucho más tiempo en la comprensión de un problema, a diferencia de los hombres.

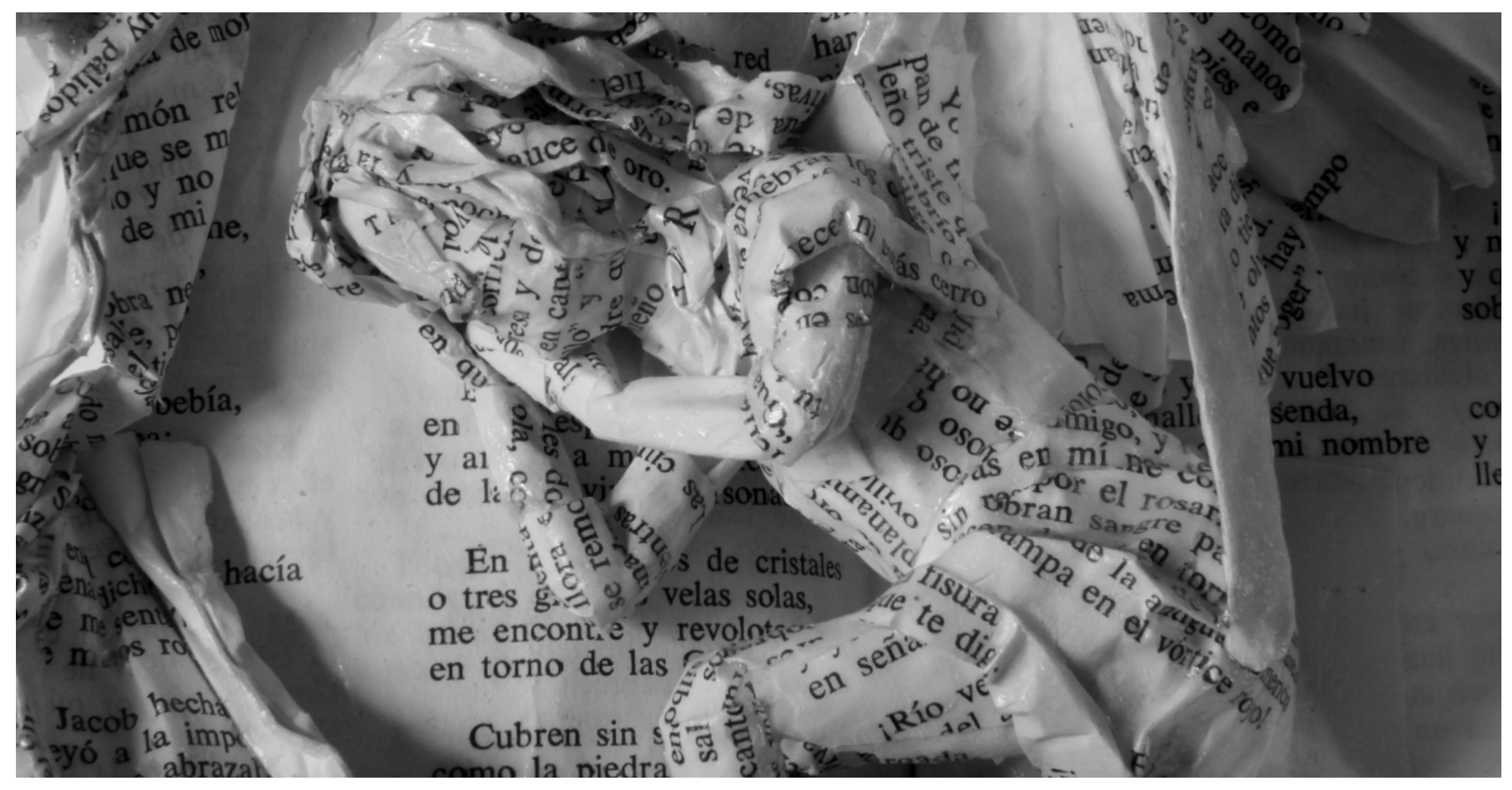

\section{Análisis correlacional}

En la siguiente tabla se muestran dos variables del eje de estilos de aprendizaje que se correlacionan positivamente con doce variables del eje resolución de problemas.

Tabla 4

Comportamiento correlacional del eje resolución de problemas en relación al eje estilos de aprendizaje

\begin{tabular}{lcc} 
& $\begin{array}{c}\text { 71.-Normalmente trato } \\
\text { de resolver los } \\
\text { problemas paso a paso }\end{array}$ & $\begin{array}{c}84 . \text { Prefiero } \\
\text { las cosas } \\
\text { estructuradas }\end{array}$ \\
\hline $\begin{array}{lc}\text { 1.- Las matemáticas son un conjunto de reglas, fórmulas y procedimientos } \\
\text { 11.- Lees el enunciado del problema }\end{array}$ & 0.18 & 0.22 \\
12.- Comprendes el problema & 0.26 & 0.17 \\
14.- Identificas los datos (lo que conoces) & 0.30 & 0.21 \\
16.- Encuentras relación entre los datos y las incógnitas & 0.32 & 0.18 \\
19. - Este problema es parecido a otros que ya resolví & 0.32 & 0.17 \\
22.- Utilizaré todos los datos del problema & 0.33 & 0.22 \\
23.- Verificas cada operación del problema & 0.23 & 0.25 \\
24.- Cuando se te presenta alguna dificultad que te deja bloqueado, & 0.31 & 0.20 \\
$\quad$ vuelves al inicio, reordenas las ideas & 0.26 & 0.17 \\
27.- Sigues un método: verbal, algebraico, gráfico, numérico & 0.37 & 0.17 \\
28.- Lees de nuevo el enunciado y compruebas que lo que se te pedía & 0.23 & 0.23 \\
$\quad$ es la solución a la que llegaste & & 0.26 \\
30.- Puedes comprobar la solución & 0.25 & 0.23 \\
\hline
\end{tabular}


Por lo que se observa en la tabla 4, se infiere que el estudiante resuelve los problemas matemáticos en relación al estilo de aprendizaje teórico. Considera que las matemáticas son un conjunto de reglas, fórmulas y procedimientos y comprende el problema, identifica y relaciona los datos con las incógnitas. Piensa que el problema es parecido a otros que ya resolvió y, si va a utilizar todos los datos, verifica cada operación.

Si se le presenta alguna dificultad, reordena las ideas, vuelve a iniciar y sigue un método: verbal, algebraico, gráfico, numérico; lee de nuevo el enunciado y comprueba la solución, potencializa las estrategias metacognitivas, el pensamiento matemático cognitivo, didáctico, epistemológico y el lenguaje matemático.

Los resultados encontrados de acuerdo al procedimiento de análisis comparativo responden a lo siguiente: Demostrar que existen diferencias de opinión entre hombres y mujeres en comprender un problema.

Se puede inferir que las mujeres le dedican mucho más tiempo en la comprensión de un problema, a diferencia de los hombres".
1.- ¿Existen diferencias significativas en comprender un problema, según el género?

$\mathrm{H}_{1}=$ Existen diferencias significativas en comprender un problema, según el género.

Por lo anterior, se concluye que las mujeres tardan más tiempo en la comprensión de un problema, a diferencia de los hombres (Ver tabla 3).
A partir del análisis estadístico correlacional se da respuesta a lo siguiente: Demostrar que la resolución de problemas, tiene relación con los estilos de aprendizaje.

2.- ¿Existe relación entre la resolución de problemas y los estilos de aprendizaje?

$\mathrm{H}_{2}=$ Existe relación entre la resolución de problemas y los estilos de aprendizaje.

Se concluye que en la resolución de problemas los alumnos piensan que estos no se pueden plantear de diferente forma, sin embargo se apoyan en seguir un método matemático (verbal, algebraico, gráfico, numérico) y tratan de resolver los problemas paso a paso, prefiriendo las cosas estructuradas (Ver tabla 4). 


\section{Referencias}

Alonso,C., Gallego, D., y Honey, P. (1997). Los estilos de aprendizaje. Ediciones Mensajero, Bilbao.

Bolívar, L. J. M., y Rojas, V. F. (2008). Los estilos de aprendizaje y el locus de control en estudiantes que inician estudios superiores y su vinculación con el rendimiento académico. Investigación y postgrado, 23(3), 199-215. Recuperado de: http://revistas.upel.edu.ve/index. php/revinpost/article/view/899/341.

Cagliolo, L., Junco, C., y Peccia, A. (2010). Investigación sobre las relaciones entre los estilos de aprendizaje y el resultado académico en las asignaturas elementos de matemática, introducción a la administración y análisis socio-económico. Revista Estilos de Aprendizaje, 3(6), 23-33. Recuperado de http://learningstyles.uvu.edu/index.php/jls/article/view/129/90.

Camarero, S. F., Martín, d. B. F., \& Herrero, D. J. (2000). Estilos y estrategias de aprendizaje en estudiantes universitarios. Psicothema, 12(4), 615-622. Recuperado de http://www. psicothema.com/pdf/380.pdf.

Escudero,I.M.,Gavilán,J.M.,y Sánchez-Matamoros,G.(2014).Una aproximación a los cambios en el discurso matemático generados en el proceso de definir. Revista latinoamericana de investigación en matemática educativa, 17(1), 7-32. Recuperado de http://www.scielo.org.mx/ scielo.php?script=sci_abstract\&pid $=$ S1665 $-24362014000100002 \&$ lng $=$ es \& nrm=iso\& tlng = es.

Felder, R. M.,y Silverman, L. K. (1988). Estilos de aprendizaje y de enseñanza en la educación de ingeniería [Versión electrónica]. Ing. Educación, 78 (7), 674-681 Disponible en: http:// www4.ncsu.edu/unity/lockers/users/f/felder/public/Papers/ LS- 1988.pdf. [Consulta: 2014, Diciembre 01].

Hernández Sampieri, R., Fernández Collado, C., y Baptista Lucio, P. (2014). Metodología de la investigación. Sexta Edición. Editorial Mc Graw Hill. México. 2014.

Ocampo, B. F., Guzmán, A.A., Camarena, G. P., y De Luna, C. R. (2014). Identificación de estilos de aprendizaje en estudiantes de ingeniería. Revista mexicana de investigación educativa, XIX(61), 401-429. Recuperado de http://www.comie.org.mx/v1/revista/portal.php?idm=es\&s ec $=S C 03 \&$ sub $=S B B \&$ \& criterio=ART61003.

Valadez, H. M. (2009). Estilos de aprendizaje y estilos de pensamiento: Precisiones conceptuales. Revista Educativa Desa, 6(11), 19-30. Recuperado de http://www.imbiomed.com. $\mathrm{mx} / 1 / 1 /$ articulos.php? method=showDetail\&id_articulo $=97099 \& i d_{\text {_ seccion }}=4504 \& i d_{\text {_ }}$ ejemplar $=9484 \&$ id_revista $=291$.

Vega-Castro, D., Molina, M., y Castro, E. (2012). Sentido estructural de estudiantes de bachillerato en tareas de simplificación de fracciones algebraicas que involucran igualdades notables. Revista latinoamericana de investigación en matemática educativa, 15(2), 233-258. Recuperado de http://www.scielo.org.mx/pdf/relime/v15n2/v15n2a5.pdf. 


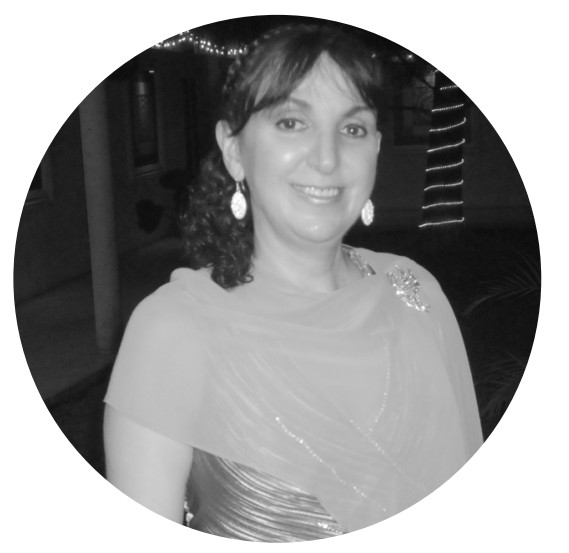

\section{Ma. del Socorro Elizondo Treviño}

Es Ingeniero Administrador de Sistemas de la Facultad de Ingeniería Mecánica y Eléctrica de la Universidad Autónoma de Nuevo León. Tiene maestría en Enseñanza de las Ciencias con Especialidad en Matemáticas de la Facultad de Filosofía y Letras y de la Facultad de Físico Matemáticas de la misma Universidad. Doctora en Ciencias de la Educación de la Facultad de Ciencia, Educación y Humanidades de la Universidad Autónoma de Coahuila. Maestra de tiempo completo de la Preparatoria 1 de la Universidad Autónoma de Nuevo León.

Correo electrónico:

socoelizondo@hotmail.com 